\title{
Hörgeräteabgabe in Seldwyla - retten, was nicht mehr zu retten ist
}

\author{
Das Bundesamt für Sozialversicherungen (BSV) liess die Audiologiekommission der \\ ORL-Gesellschaft neue Richtlinien für ein Pauschalsystem bei der Hörgeräteanpassung \\ erstellen. Es stellte sich aber heraus, dass vom BSV hinter verschlossenen Türen bereits \\ eine einfache und billige Pauschallösung erarbeitet worden war. Auf die Vorgabe der \\ Anpassung der Geräte durch ausgebildete Akustiker wird nun gänzlich verzichtet.
}

\section{Thomas Linder}

Chefarzt, Klinik für Hals-NasenOhren-, Hals- und Gesichtschirurgie, Luzerner Kantonsspital Luzern

Auf Seite 1130 nimmt das BSV zu diesem Beitrag Stellung.
1 Bertoli S, Bodmer D, Probst Bertoli R. Survey on hearing aid outcome in Switzerland: associations with type of fitting (bilateral/unilateral), level of hearing aid signal processing, and hearing loss. Int. J Audiol. 2010;49:333-46.

Korrespondenz:

Prof. Dr. Thomas Linder Luzerner Kantonsspital Spitalstrasse

CH-6000 Luzern

Tel. 0412054951

thomas.linder@ksl.ch
Wäre es für alle Beteiligten nicht bitter ernst, man könnte die folgende Geschichte als kurioses Lehrstück in der Schweizer Gesundheitspolitik werten und lediglich schmunzeln. Es droht jedoch Ähnliches wie beim Praxislabor oder bei der direkten Medikamentenabgabe: Bundesbern verordnet, und wir Ärzte schlucken die Kröte. Und wohl ist es schon zu spät.

\section{Höchste Tragerate und Zufriedenheit der Hörgeräteträger}

Während Jahrzehnten hat sich ein System der Hörgeräteabgabe in der Schweiz entwickelt, das es dem Patienten unter der Vorgabe einer einfachen und zweckmässigen Versorgung vonseiten der Kostenträger (IV und AHV) ermöglicht hat, zuzahlungsfrei oder mit freiwilliger Zuzahlung eine bestmögliche Versorgung zu erhalten, die ihrerseits wieder durch eine Schlusskontrolle des Arztes bestätigt (oder zur Verbesserung zurückgewiesen) wurde. Dazu wurden von der ORL-Fachgesellschaft Richtlinien für Experten erarbeitet und kontrolliert, die Audiometrie-Kabinen mussten jährlich gewartet und von unabhängigen Messexperten überprüft werden, die Anforderungen zur Festlegung des Ausmasses der Schwerhörigkeit wurden fortwährend in der Audiologiekommission (AK) in Fronarbeit neu definiert (sowohl nach audiologischen als auch nach sozial-emotionalen und beruflichen Kriterien), und die Anpassung der Hörgeräte durfte nur von anerkannten und ausgebildeten Hörgeräteakustikern vorgenommen werden. Eine Studie von Bertoli, Probst et al. [1] belegte denn auch die im europäischen Vergleich höchste Tragerate und Zufriedenheit der Hörgeräteträgerinnen und -träger.

\section{Sparzwang}

Eine weitere Studie der Eidgenössischen Finanzkontrolle (EFK) von 2007 sah jedoch auch ein Sparpotential zugunsten der Sozialversicherung, insbesondere durch die im europäischen Vergleich sehr hohen Gerätekosten, und tatsächlich schienen die Akustikergeschäfte in der Schweiz zu boomen, und zahlreiche deutsche Ketten drangen in den Schweizer Markt ein. Der generelle Sparauftrag an die IV/AHV machte logi-

\section{Appareils auditifs à Seldwyla - sauver ce qui n'est plus à sauver}

Il y a encore une année, le directeur de l'OFAS rassurait la Commission d'Audiologie de la Société d'ORL en lui promettant que le système alors en vigueur, qui prévoyait que les appareils auditifs soient réglés et examinés par des experts, serait maintenu. Tandis que nous, médecins ORL, envoyions nos assistantes médicales à des ateliers de formation - respectant ainsi les directives de I'OFAS - et pendant que les acousticiens faisaient du lobbying contre les personnes concernées au Parlement respectivement pour ou contre la centralisation de I'achat d'appareils auditifs, les collaborateurs de I'OFAS continuaient à participer aux séances de la Commission tout en élaborant en secret une solution forfaitaire bon marché. Conviées en dernière minute à une séance de discussion, les personnes directement concernées (médecins ORL, acousticiens et pro audito Suisse) n'ont pu que constater que leur position de refus fondé avait été ignorée. En effet, les brochures d'information sur le nouveau système forfaitaire étaient déjà imprimées dans toutes les langues nationales. Nous pouvons ainsi remercier le conseiller fédéral Didier Burkhalter et ses conseillers, grâce à qui un système éprouvé depuis des décennies ainsi qu'un suivi efficace ayant fait ses preuves pour l'ajustage d'appareils auditifs a été remplacé par une solution de remboursement forfaitaire rudimentaire. Celle-ci encourage de surcroît, sur conseils de la BSV, les malentendants à acheter leurs appareils à un tarif inférieur également à l'étranger et cela sans conseils d'un spécialiste. Ils constateront bien ce que vaut I'appareil qu'ils ont acheté en le portant. Triste, mais vrai... 


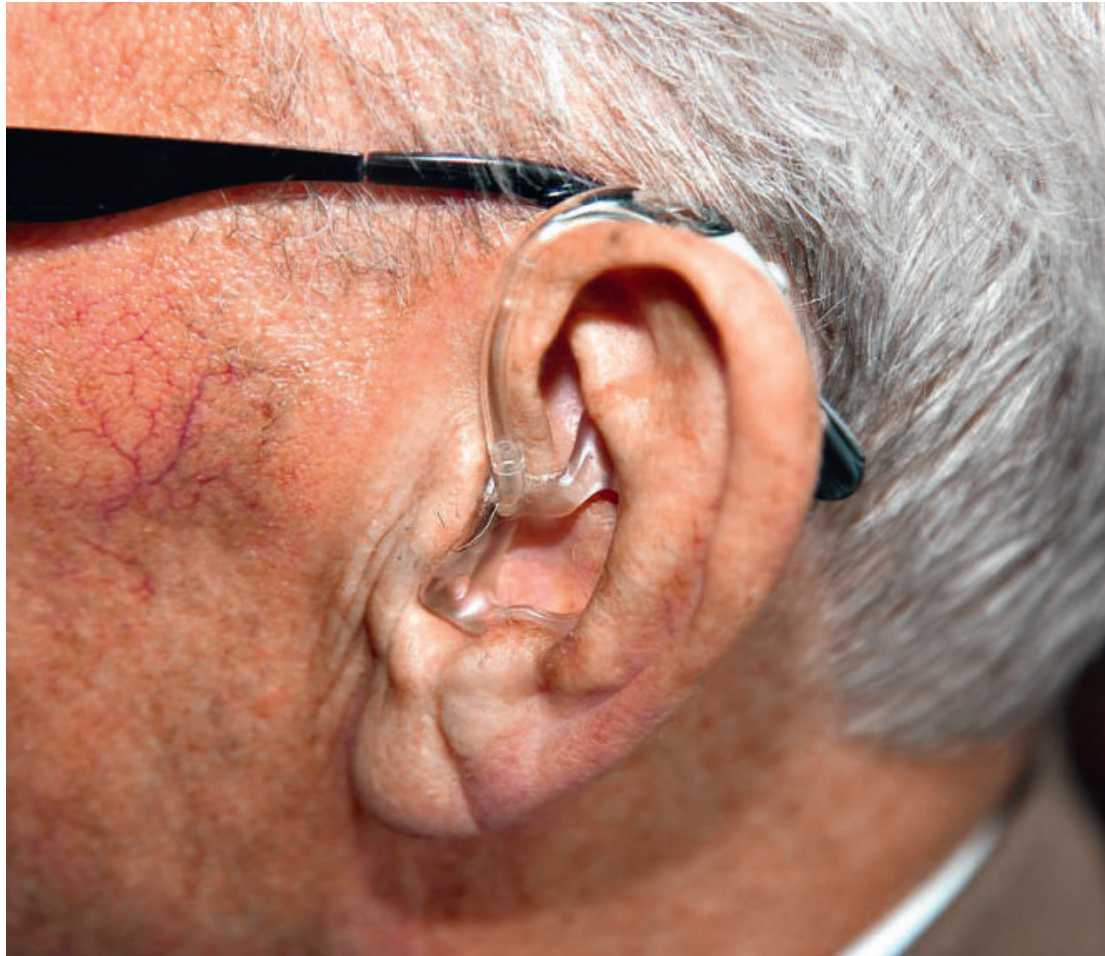

Hörgeräte müssen den individuellen Bedürfnissen des Hörbehinderten angepasst werden: Sie werden aktiv programmiert, auf individuelle Frequenzen abgestimmt und gegen zu starke Verstärkung limitiert. geräten durch das BSV, in der redlichen Hoffnung, dass das Sparziel primär bei der Industrie seine Wirkung zeigen würde und nicht bei den direkt Betroffenen. Während sich nun also Patientenvertreter und Hersteller/Verkäufer gegenseitig und auch untereinander zerstritten und debattierten, planten die Spitzen des BSV eine ganz andere Lösung. Die Idee des zentralen Einkaufs war in der Praxis gar nicht umsetzbar, in Bern sind keine Lagerräume für neue und ausgediente Hörgeräte verfügbar, und keine BSV-Lieferwagen oder Pizza-Kuriere stehen für eine mögliche Verteilung zur Verfügung.

\section{Heimliche Abkehr vom Expertisensystem}

Das BSV hatte schon längst eine Fachperson gefunden, die nun in akribischer Arbeit neue Kriterien testete, um mit ganz wenigen Hörtests eine neue Einteilung zu zementieren. Berufliche oder sozial-emotionale Kriterien sollten gänzlich weggelassen werden. Schliesslich braucht ein Brillenrezept auch nur eine Visusbestimmung, entsprechend könnte ein Hörgerät ja auch nur eine einzige Hörschwelle als Ausgangswert erfordern. Die Mitarbeiter des BSV sassen weiterhin gut gelaunt mit den ORL-Ärzten an den offiziellen gemeinsamen Sitzungen zusammen, sprachen von zunehmendem Spardruck durch das Parlament und stellten baldige Änderungen in der Hörgeräteabgabe in Aussicht. Mehrfach wurde die AudiologieKommission (AK) angefragt, ob sie denn bereit wäre, neue Kriterien zu definieren oder die jetzigen etwas zu verfeinern. Der eigentliche Auftrag wurde jedoch vorerst nicht erteilt, denn im Hintergrund arbeitete ja der pensionierte Kollege, und weitere Mitarbeiter des BSV hatten genügend Zeit, sich im Ausland über andere Modelle zu informieren. Der Entscheid, eine einfache, billige Pauschallösung einzuführen, war äusserst verlockend. Tunlichst meldeten sich auch Rollstuhlfahrer zu Wort, die eine pauschale Auszahlung für ihre Hilfsmittel engagiert verteidigen, da sie ja als körperlich Behinderte genügend Selbstbestimmung und Autonomie haben, sich nach Auszahlung eines Betrages das richtige Gefährt auszusuchen. Im Trubel der Ereignisse war es der Führung des BSV entgangen, dass Hörgeräte keine Brillen und kein Rollmaterial sind. Während Brillen passiv durch Brechung die Sehkraft verbessern und ein klemmender Rollstuhl nicht mehr geradeaus fährt, müssen Hörgeräte aktiv programmiert, auf individuelle Frequenzen abgestimmt, gegen zu starke Verstärkung limitiert und auf die individuellen Bedürfnisse des Hörbehinderten angeglichen werden.

Noch im Juli 2010 beruhigte der Direktor des BSV, Herr Rossier, die AK und versprach, das Expertisensystem zu erhalten. Er lobte die konstruktive Zusammenarbeit mit der Kommission und äusserte sein Wohlwollen bezüglich der gemeinsamen Erstellung neuer Richtlinien. Im Oktober wurde endlich der AK ein detaillierter Auftrag des BSV zur Erstellung neuer Richtlinien im Hinblick auf ein Pauschalsystem erteilt. Vier Wochen später hatte die Kommission einen 
ersten Entwurf fristgerecht abgegeben. Kurz nach dem Samichlaustag lud das BSV die Akustiker, pro audito schweiz (Vertreter der Hörbehinderten) und die Präsidentin der AK zu einer wichtigen Besprechung ein. Auf die eingereichten Vorschläge der AK wurde gar nicht mehr eingegangen, sondern eine Pauschallösung präsentiert, bei der dem Hörbehinderten ein fixer Betrag (im IV-Alter 840 Franken für ein Hörgerät, bzw. 1650 Franken für zwei Geräte) ausbezahlt wird, der sowohl die Gerätekosten als auch die Anpasskosten abdecken sollte. Auf die bisherige Vorgabe der Anpassung der Geräte durch ausgebildete Akustiker wird gänzlich verzichtet, ja ein günstiger Einkauf jenseits der Schweizer Grenze sogar aktiv von der Bundesbehörde empfohlen. Da es die Beamten gewohnt sind, auch weiterhin detailliert Tabellen zu führen, verlangte das BSV, dass auf der Rechnung u.a. die genaue Bezeichnung des angeschafften Hörgerätes mit der vom Bundesamt für Metrologie zugeteilten Num- rigen ärztlichen Tätigkeit kraftvoll aufzutreten. Nur hatte leider der FMH-Präsident für unser Anliegen kein Gehör, bzw. war anderweitig beschäftig.

Während das BSV bereits Broschüren in allen Landessprachen druckte, um die Versicherten (also unsere hörbehinderten Patienten) über diesen genialen Systemwechsel zu informieren, gewährten uns die Chefs des BSV nochmals die Möglichkeit der «Aussprache». Auf Kritik der Ärzte wurde jedoch ungehalten reagiert, $\operatorname{man}(\mathrm{n})$ war betupft und beleidigt, und die Sitzung wurde vorzeitig abgebrochen. Nun kann man immerhin vor den Medien behaupten, man hätte ja die ORL-Ärzte jederzeit informiert. Gleichwohl liess es sich Bundesrat Burkhalter nicht nehmen, auch die Behindertenvertreter zum Gespräch einzuladen. In der natürlich kurzen Besprechungszeit des vielbeschäftigen Bundesrates wurden denn auch der Vizepräsident und der Geschäftsleiter von pro audito schweiz gemassregelt, wie es denn den Behin-

\section{Protestierend, kopfschüttelnd und aufgewühlt verliessen die einge- ladenen Präsidenten der Akustiker, von pro audito schweiz und der ORL-Gesellschaft die Sitzung des BSV.}

mer (Zitat BSV) aufgeführt ist. Da das Bundesamt für Messwesen jedoch in der EU (noch) keine Vorschriften erlassen kann, darf der Beamte in Bern für Geräte aus dem Ausland (z. B. Kosovo) lediglich die Bezeichnung des verkauften Gerätes in seine Tabelle eintragen und dann auch die Pauschale auszahlen. Protestierend, kopfschüttelnd und aufgewühlt verliessen die eingeladenen Präsidenten der Akustiker, pro audito schweiz und der ORL-Gesellschaft die Sitzung des BSV.

Doch noch bevor sie die Ausgangstüre erreicht hatten, war die Pressemitteilung des BSV über die neuerarbeitete einzigartige Pauschallösung mittels Mausklick verschickt worden und die verdutzten Präsidenten konnten bereits ersten Journalisten Red und Antwort stehen. Leider war das Interesse der schreibenden Zunft kurz vor Weihnachten nicht besonders gross, so dass der Systemwechsel in den Printmedien kaum wahrgenommen wurde. Da das BSV zudem auch noch auf die Schlusskontrolle der Hörgeräteanpassung (bisherige Schlussexpertise) vollständig verzichtete, aber mehrfach auf den Referenzmarkt Deutschland hinwies (z.B. bei der Berechnung der Pauschalen, die in Deutschland tatsächlich kleiner sind), waren wir ORL-Ärzte besonders gefordert. Denn auch in Deutschland erfolgt die Hörgeräteanpassung durch ausgebildete Akustiker und wird schliesslich durch den ORL-Arzt mittels Begutachtung und Endabnahme kontrolliert. Der Vorstand unserer Gesellschaft und die Präsidentin wollten umgehend beim FMH-Präsidenten vorstellig werden, um gegen diese unerhörten Einschnitte in unserer bishe- derten überhaupt in den Sinn kommen könnte, sich erst noch öffentlich gegen seine Verordnung zu wehren. Ja, wann und wie eigentlich soll man denn noch protestieren können, wenn die bundesrätliche Verordnung in diesen Tagen umgesetzt werden sollte zur Einführung im Juli?

Als Ohrenärzte kennen wir das Schicksal und die Bedürfnisse von Hörbehinderten durch unsere tägliche Arbeit. Wir haben in Klinik und Praxis Tausende dieser Betroffenen persönlich betreut und viele dieser Menschen über mehr als 20 Jahre lang begleitet. Die Audiologie-Kommission der Schweizerischen ORLGesellschaft setzte sich seit Jahren dafür ein, dass die Qualität der Hörgeräteversorgung in der Schweiz einen hohen Stand erreicht hat und die Ausbildung der Ohrenärztinnen und Ohrenärzte und ihrer medizinischen Hilfspersonen in diesem Bereich hervorragend ist. Die Art und Weise, wie das BSV unter dem Druck politischer Vorgaben aus dem EDI die fundierten Einwände der Fachleute in den Wind geschlagen hat und einen Systemwechsel bei der Hörgeräteabgabe erzwingt, hinterlässt ein Gefühl der Ohnmacht. Noch selten waren sich die direkt Betroffenen (sämtliche Verbände der Hörbehinderten), die Hersteller (alle Verbände der Akustiker) und wir Ärzte (Schweizerische ORL-Gesellschaft) in allen Punkten einig: So darf es nicht kommen. Aber eben, Seldwyla tickt anders: FDP-Bundesrat Burkhalter und seine Berater werden sich entgegen aller fachlichen und menschlichen Argumente durchsetzen. Als Trost bleibt: Am Wahltag ist Zahltag. 